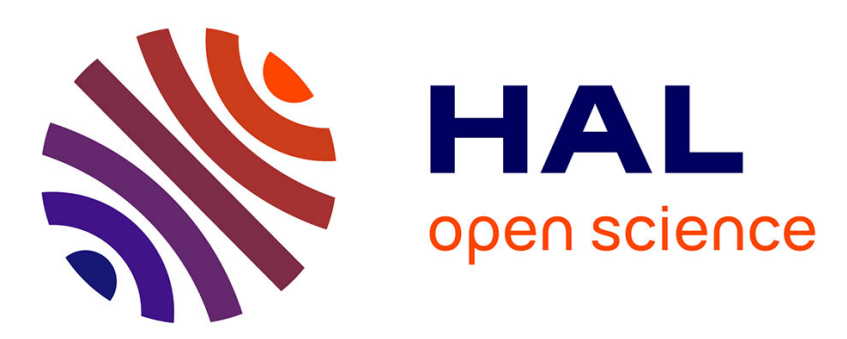

\title{
Numerical study of the regularizing effect of the 3D weakly transverse BBM equations for long times Youcef Mammeri
}

\section{To cite this version:}

Youcef Mammeri. Numerical study of the regularizing effect of the 3D weakly transverse BBM equations for long times. Applied Mathematics and Computation, 2013, 219, pp.5162 - 5173. 10.1016/j.amc.2012.10.112 . hal-01090305

\section{HAL Id: hal-01090305 https://hal.science/hal-01090305}

Submitted on 3 Dec 2014

HAL is a multi-disciplinary open access archive for the deposit and dissemination of scientific research documents, whether they are published or not. The documents may come from teaching and research institutions in France or abroad, or from public or private research centers.
L'archive ouverte pluridisciplinaire HAL, est destinée au dépôt et à la diffusion de documents scientifiques de niveau recherche, publiés ou non, émanant des établissements d'enseignement et de recherche français ou étrangers, des laboratoires publics ou privés. 


\title{
Numerical study of the regularizing effect of the 3D weakly transverse BBM equations for long times
}

\author{
Y. Mammeri \\ Laboratoire Amiénois de Mathématique Fondamentale et Appliquée, \\ CNRS UMR 7352, Université de Picardie Jules Verne, \\ 80039 Amiens, France. \\ Email: youcef.mammeri@u-picardie.fr
}

October 2012

\section{Contents}

1 Numerical discretizations 3

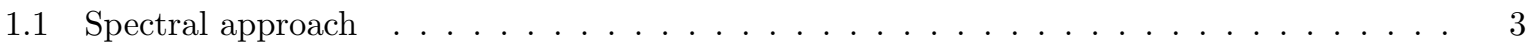

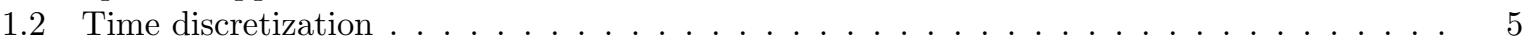

2 Long time behavior for localized initial data $\quad 6$

2.1 Non-generalized case: $p=1 \ldots \ldots \ldots \ldots \ldots \ldots$

2.2 Generalized case: $p>1 \ldots \ldots \ldots \ldots \ldots$

3 Transverse instabilities in the $y$ and $z$-directions $\quad 9$

3.1 Non-generalized case: $p=1 \ldots \ldots \ldots \ldots \ldots$

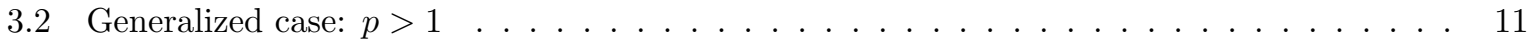

4 Transverse instability in the $z$-direction $\quad 12$

Abstract. From a spectral method combined with a predictor-corrector scheme, we numerically study the behavior in time of solutions of the three-dimensional generalized Kadomtsev-Petviashvili equations regularized using the BBM trick. The solution no longer blows up and the solitonic behavior is observed.

Keywords. 3D-KP equations, BBM equation, spectral method, predictor-corrector scheme, dispersion, solitonic behavior, transverse instability

MS Codes. 35B05, 35B35, 35Q53, 65L05, 65M70

\section{Introduction}

The Kadomtsev-Petviashvili equations (KP) in three-dimensional space

$$
u_{t}+u_{x}+u u_{x}+u_{x x x}+a \partial_{x}^{-1} u_{y y}+b \partial_{x}^{-1} u_{z z}=0
$$

is used to describe the Bose-Einstein condensation [5], or electromagnetic waves in a ferromagnetic medium [14]. Here $\partial_{x}^{-1}$ denotes the anti-derivative, defined such that $\widehat{\partial_{x}^{-1} u}\left(\xi_{1}, \xi_{2}, \xi_{3}\right):=\widehat{u}\left(\xi_{1}, \xi_{2}, \xi_{3}\right) /\left(\mathrm{i} \xi_{1}\right)$, and $\widehat{u}$ represents the Fourier transform of $u$, the constants $a, b$ are normalized to \pm 1 . 
Using the Benjamin-Bona-Mahony trick [3] (the change of variables $\varepsilon^{-1} u\left(\varepsilon^{-1 / 2} x, \varepsilon^{-1} y, \varepsilon^{-1} z, \varepsilon^{-1 / 2} t\right.$ ) is applied, with $\varepsilon$ small), we can replace $u_{x}$ by $-u_{t}+O(\varepsilon)$ in (1) to find the so-called regularized KP equations (or the BBM equation)

$$
u_{t}+u_{x}+u u_{x}-u_{x x t}+a \partial_{x}^{-1} u_{y y}+b \partial_{x}^{-1} u_{z z}=O(\varepsilon) .
$$

In this paper, we are concerned with the regularized generalized KP equations in three-dimensional space

$$
u_{t}+u_{x}+u^{p} u_{x}-u_{x x t}+a \partial_{x}^{-1} u_{y y}+b \partial_{x}^{-1} u_{z z}=0
$$

where $p \geq 1$.

Recall that the mass

$$
\int_{\mathbb{R}^{3}} u^{2}(x, y, z, t)+u_{x}^{2}(x, y, z, t) d x d y d z
$$

is conserved by the flow associated with (3). Some theoretical results concerning the behavior of solutions of (1) have been recently established, in particular the existence of solitary waves $[6,7]$ and the blow-up in finite time $[15,16,24]$. Many theoritical and numerical works related with Kadomtsev-Petviashvili equations and its regularized version in dimension two can be found in the literature $([4,8,11,13,24,25$, $27,28]$ and references therein). Few literatures are concerning the dimension three, therefore numerical simulations are of interest $[12,17,26,30,32]$. We recently studied the numerical behavior of the solutions of (1), and observed three phenomenons : the blow-up in finite time, the dispersion and the solitonic behavior [10].

The aim of this paper consists in inspecting numerically the behavior of the solution of (3). We observe the regularizing effect of (3): the blow-up in finite time of solutions does no longer occur.

This paper is subdivided into four sections. In Section 1, we discretize the Cauchy problem associated with (3), by combining a spectral method for the space discretization and a predictor-corrector scheme for the time discretization. Sections 2, 3 and 4 systematically deal with numerical simulations.

We study in Section 2 the propagation, based on (3), of localized initial data. The numerical inspections show in the contexts where $p \geq 1, a=b=-1$, and $p \geq 3, a=b=1$ or $a b=-1$, the $l^{\infty}$-norm of the discrete solution of (3) is first perturbed and then is stabilized. This is defined as the solitonic behavior. This state, for which the amplitude of the wave remains constant, is a intermediary between the dispersion due to the linearity (the amplitude of the wave decreases and the wave is broken down) and the blow-up in finite time due to the non-linearity. Furthermore, soliton is a particular solution which is translated at a constant velocity without loss of shape. Concerning (1), the numerical solutions blow up in finite time in the same contexts [10].

In the two next sections, we are concerned with the numerical investigation of the transverse instabilities, we aim to characterize the unstable modes [1, 2, 19, 20, 21, 22, 23, 29]. The unstable modes are obtained from the linearized equation around $\Phi_{c}$, a solitary wave, as

$$
u(x, y, t)=\Phi_{c}(x-c t)+\varepsilon \mathrm{e}^{\sigma t+\mathrm{i} \frac{2 \pi y}{\lambda y}} \widetilde{u}(x),
$$

with $\lambda_{y}>0, \operatorname{Re}(\sigma)>0, \widetilde{u} \in L^{2}(\mathbb{R})$. The line-soliton

$$
\Phi_{c}(x)=\left(\frac{(p+1)(p+2)}{2} c\right)^{1 / p} \operatorname{sech}^{2 / p}\left(\frac{p}{2} x\right),
$$

is considered in Section 3 and we study the solution of (3) with the initial datum:

$$
u_{0}(x, y, z):=\Phi_{c(y, z)}(x),
$$

where $c(y, z):=c\left(1+\varepsilon \cos \left(2 \pi y / \lambda_{y}+2 \pi z / \lambda_{z}\right)\right)$ is a perturbation of the velocity of the line-soliton, in an infinitesimal way in the $y$ and $z$-directions, $\lambda_{y}, \lambda_{z}>0$, and $\varepsilon>0$ is a fixed small value. 
When $p \geq 2, a=b=-1$, or $a b=-1$, there exist two critical transverse wavelengths $0<\lambda_{c}^{1} \ll \lambda_{c}^{2}$, depending only on $c$, such that the discrete solution of (3) with such an initial datum $u_{0}$ is unstable under the flow of (3) if $\lambda_{c}^{1}<\lambda_{y}<\lambda_{c}^{2}$ or $\lambda_{c}^{1}<\lambda_{z}<\lambda_{c}^{2}$.

The study of the transverse instability in the $z$-direction is done in Section 4 , by considering as initial datum the profile of the Zaitsev traveling waves [31], which exists for $p=1$ and $a=-1$ :

$$
\begin{gathered}
u_{0}(x, y, z):=\psi_{c(z)}(x, y), \\
\psi_{c}(x, y)=12 \alpha^{2} c \frac{1-\beta \cosh (\alpha x) \cos (\delta y)}{(\cosh (\alpha x)-\beta \cos (\delta y))^{2}}, \beta=\sqrt{1-\frac{3 c \alpha^{4}}{\delta^{2}}}, 3 c \alpha^{4}<\delta^{2}, \alpha^{2}+\frac{\delta^{2}}{c \alpha^{2}}=1 .
\end{gathered}
$$

The velocity is here perturbed in the $z$-direction; $c(z):=c\left(1+\varepsilon \cos \left(2 \pi z / \lambda_{z}\right)\right), \lambda_{z}>0$, and $\varepsilon>0$ a fixed small value. The wavelength $\lambda_{z}$ translates the instability by transverse perturbations in the sense that, when $b=-1$, there exist two critical transverse wavelengths $0<\lambda_{c}^{1} \ll \lambda_{c}^{2}$, depending only on $c$, such that the solution of (3) with $u_{0}$ as initial datum behaves like a soliton, not close to the initial datum if $\lambda_{c}^{1}<\lambda_{z}<\lambda_{c}^{2}$. The solution is hence unstable.

\section{Numerical discretizations}

Recall that the initial value problem is globally in time well-posed in dimension two $[4,25]$ :

Theorem 1.1 [25] There exists a functional space continuously $\mathcal{X}$ embedded in $\mathcal{C}(\mathbb{R} ; H)$, with $H \subset$ $L^{2}\left(\mathbb{R}^{2}\right)$ equipped with the norm

$$
\|u\|_{H}=\left(\|u\|_{L^{2}}^{2}+\left\|u_{x}\right\|_{L^{2}}^{2}\right)^{1 / 2},
$$

such that for any $u_{0} \in H$, there exists a unique global in time solution $u \in \mathcal{X}$ with $u_{0}$ as initial datum.

In [4], the global well-posedness is provided in the space

$$
\left\{u, u_{x}, u_{y}, u_{x x}, \partial_{x}^{-1} u_{y} \in L^{2}\left(\mathbb{R}^{2}\right)\right\} .
$$

Let us consider the Cauchy problem based on (3), where $u_{0}$ denotes the initial datum:

$$
u(x, y, z, 0):=u_{0}(x, y, z),
$$

with the following functional settings:

$$
\left\{u, u_{x}, u_{y}, u_{z}, u_{x x}, u_{y y}, u_{z z}, \partial_{x}^{-1} u_{y}, \partial_{x}^{-1} u_{z}, \partial_{x}^{-2} u_{y y}, \partial_{x}^{-2} u_{z z} \in L^{2}\left(\mathbb{R}^{3}\right)\right\} .
$$

By combining a spectral approach with a predictor-corrector scheme, we discretize here the Cauchy problem based on (3).

\subsection{Spectral approach}

Spectral methods are very suitable to the discretization of equations such as (3), the non-linearity and the anti-derivative appearing in (3) being easily treated.

For $\left(\xi_{1}, \xi_{2}, \xi_{3}\right) \in \mathbb{R}^{3}$, and $t \geq 0$, we use the notation

$$
\widehat{u}\left(\xi_{1}, \xi_{2}, \xi_{3}, t\right):=\int_{-\infty}^{\infty} \int_{-\infty}^{\infty} \int_{-\infty}^{\infty} u(x, y, z, t) \mathrm{e}^{-\mathrm{i}\left(\xi_{1} x+\xi_{2} y+\xi_{3} z\right)} d x d y d z .
$$


By applying this Fourier transform to the considered Cauchy problem, it follows that the singularity for $\xi_{1}=0$ needs to be overcame. Looking moreover for solutions of (3) such that $u, u^{p+1}$ and $u_{t} \in$ $L^{1}\left(\mathbb{R}^{3}\right) \cap L^{2}\left(\mathbb{R}^{3}\right)$, we notice that $\forall\left(\xi_{2}, \xi_{3}\right) \in \mathbb{R}^{2}, \forall t>0$,

$$
\lim _{\xi_{1} \longrightarrow 0} \widehat{u}\left(\xi_{1}, \xi_{2}, \xi_{3}, t\right)=0, \quad \lim _{\xi_{1} \longrightarrow 0} \frac{\widehat{u}\left(\xi_{1}, \xi_{2}, \xi_{3}, t\right)}{\xi_{1}}=0,
$$

provided that

$$
\widehat{u}_{0}\left(0, \xi_{2}, \xi_{3}\right)=0, \quad \lim _{\xi_{1} \longrightarrow 0} \frac{\widehat{u_{0}}\left(\xi_{1}, \xi_{2}, \xi_{3}\right)}{\xi_{1}}=0 .
$$

Indeed, by integrating over $\mathbb{R}$ with respect to $x$, we have

$$
\int_{-\infty}^{+\infty} u_{y y}(x, y, z, t)+u_{z z}(x, y, z, t) d x=0
$$

Since $u$ vanishes at infinity, we obtain for all time $t \in \mathbb{R}$

$$
\int_{-\infty}^{+\infty} u(x, y, z, t) d x=0=\int_{-\infty}^{+\infty} u_{0}(x, y, z) d x
$$

Therefore, integrate over $\mathbb{R}$ with respect to $x$ provides

$$
\int_{-\infty}^{+\infty} \partial_{x}^{-1} u_{y y}(x, y, z, t)+\partial_{x}^{-1} u_{z z}(x, y, z, t) d x=0, \text { and } \int_{-\infty}^{+\infty} \partial_{x}^{-1} u(x, y, z, t) d x=0 .
$$

In view of numerical computations in a bounded and periodised domain $\Omega$, we now consider (3) in $[-K, K] \times[-L, L] \times[-M, M]=: \Omega$, where $K, L, M>0$ are fixed values allowing us to express the relations (4) as below

$$
\left\{\begin{aligned}
& \int_{-M}^{M} \int_{-L}^{L} \mathrm{e}^{-\mathrm{i}\left(\xi_{2} y+\xi_{3} z\right)}\left(\int_{-K}^{K} u_{0}(x, y, z) d x\right) d y d z=0 \\
& \int_{-M}^{M} \int_{-L}^{L} \mathrm{e}^{-\mathrm{i}\left(\xi_{2} y+\xi_{3} z\right)}\left(\int_{-K}^{K} \int_{-K}^{x} u_{0}\left(x_{1}, y, z\right) d x_{1} d x\right) d y d z=0 .
\end{aligned}\right.
$$

Let us consider now the discrete Fourier basis consisting of trigonometric polynomial functions

$$
\mathrm{e}^{\mathrm{i} k \frac{\pi}{K} x} \mathrm{e}^{\mathrm{i} l \frac{\pi}{L} y} \mathrm{e}^{\mathrm{i} m \frac{\pi}{M} z} ; \quad \mathrm{i}^{2}=-1, \quad-\frac{N_{x}}{2} \leq k \leq \frac{N_{x}}{2}-1, \quad-\frac{N_{y}}{2} \leq l \leq \frac{N_{y}}{2}-1, \quad-\frac{N_{z}}{2} \leq m \leq \frac{N_{z}}{2}-1,
$$

where $N_{x}, N_{y}, N_{z} \in 2 \mathbb{N}^{\star}$ represent the numbers of modes. Let us set $N_{x}=2 K / \Delta x, N_{y}=2 L / \Delta y$ and $N_{z}=2 M / \Delta z$, where $\Delta x, \Delta y, \Delta z>0$. For any non periodic scalar function $u$, locally summable, we denote by $u_{K, L, M}$ the function equal to $u$ in $\Omega$ and extended outside of $\Omega$ as a periodic function of period equal to $2 K$ in the $x$-direction, $2 L$ in the $y$-direction, $2 M$ in the $z$-direction. The expression of $u_{K, L, M}$ in the considered discrete basis is as follows,

$$
u_{K, L, M}(x, y, z)=\sum_{k=-\frac{N_{x}}{2}, l=-\frac{N_{y}}{2}, m=-\frac{N_{z}}{2}}^{\frac{N_{x}}{2}-1, \frac{N_{y}}{2}-1, \frac{N_{z}}{2}-1} \widehat{u}\left(k \frac{\pi}{K}, l \frac{\pi}{L}, m \frac{\pi}{M}\right) \mathrm{e}^{\mathrm{i} k \frac{\pi}{K} x} \mathrm{e}^{\mathrm{i} l \frac{\pi}{L} y} \mathrm{e}^{\mathrm{i} m \frac{\pi}{M} z}
$$

with

$$
\widehat{u}\left(k \frac{\pi}{K}, l \frac{\pi}{L}, m \frac{\pi}{M}\right)=\frac{1}{(2 K)(2 L)(2 M)} \int_{-K}^{K} \int_{-L}^{L} \int_{-M}^{M} u(x, y, z) \mathrm{e}^{-\mathrm{i} k \frac{\pi}{K} x} \mathrm{e}^{-\mathrm{i} l \frac{\pi}{L} y} \mathrm{e}^{-\mathrm{i} m \frac{\pi}{M} z} d x d y d z .
$$


We are then concerned with a system of $N_{x} \times N_{y} \times N_{z}$ ordinary differential equations and $N_{x} \times N_{y} \times N_{z}$ unknowns: for each triplet $\left(\xi_{1}, \xi_{2}, \xi_{3}\right)$, find $\widehat{u}\left(\xi_{1}, \xi_{2}, \xi_{3}, t\right)$ satisfying

$$
\left\{\begin{array}{l}
\left(1+\xi_{1}^{2}\right) \widehat{u}_{t}\left(\xi_{1}, \xi_{2}, \xi_{3}, t\right)+\mathrm{i} \xi_{1} \frac{\widehat{u^{p+1}}}{p+1}\left(\xi_{1}, \xi_{2}, \xi_{3}, t\right)+\mathrm{i}\left(\xi_{1}+a \xi_{2}^{2} / \xi_{1}+b \xi_{3}^{2} / \xi_{1}\right) \widehat{u}\left(\xi_{1}, \xi_{2}, \xi_{3}, t\right)=0, \quad \forall t>0 \\
\widehat{u}\left(0, \xi_{2}, \xi_{3}, t\right)=\widehat{u}_{0}\left(0, \xi_{2}, \xi_{3}\right), \quad \forall t>0, \\
\widehat{u}\left(\xi_{1}, \xi_{2}, \xi_{3}, 0\right)=\widehat{u}_{0}\left(\xi_{1}, \xi_{2}, \xi_{3}\right)
\end{array}\right.
$$

where

$$
\left\{\begin{array}{l}
\xi_{1}=k \frac{\pi}{K}, \xi_{2}=l \frac{\pi}{L}, \xi_{3}=m \frac{\pi}{M}, \\
-\frac{N_{x}}{2} \leq k \leq \frac{N_{x}}{2}-1, \quad-\frac{N_{y}}{2} \leq l \leq \frac{N_{y}}{2}-1, \quad-\frac{N_{z}}{2} \leq m \leq \frac{N_{z}}{2}-1,
\end{array}\right.
$$

and the datum $u_{0}$ is subject to (5).

\subsection{Time discretization}

In view of efficient numerical computations on long time intervals, we consider predictor-corrector method [18]. Also, in view of flexible numerical implementations, we restrict ourselves to one-step schemes.

Let $\Delta t>0$ be the step of the time discretization, and set $t_{n}=n \Delta t$. For $n \in \mathbb{N}$, we denote by $\widehat{u}_{n}$ an approximation of $\widehat{u}\left(\xi_{1}, \xi_{2}, \xi_{3}, t_{n}\right)$ and by $u_{n}$ an approximation of $u\left(x, y, z, t_{n}\right)$, where $(x, y, z) \in \Omega$. The method we consider for solving (6) - (7) consists of combining the explicit Euler method for the predictor and the Crank-Nicolson method for the corrector. The resulting scheme is a predictor-corrector method and is of order two with respect to the time step [18] under suitable regularities of the solution of (6) (7).

By denoting by $m \geq 1$ a maximal number of iterations, the algorithm considered for solving (6) - (7) is described as follows:

- Set $\widehat{u}_{0, m}:=\ldots:=\widehat{u}_{0,1}:=\widehat{u}_{0,0}:=\widehat{u}_{0}$.

- For $n=0,1, \ldots$, compute:

$$
\widehat{u}_{n+1,0}:=\widehat{u}_{n, m}-\frac{\mathrm{i} \Delta t}{1+\xi_{1}^{2}}\left(\xi_{1} \frac{\widehat{u_{n, m}^{p+1}}}{p+1}+\left(\xi_{1}+a \xi_{2}^{2} / \xi_{1}+b \xi_{3}^{2} / \xi_{1}\right) \widehat{u}_{n, m}\right),
$$

- For $r=0,1, \ldots, m-1$,

$$
\begin{array}{r}
\widehat{u}_{n+1, r+1}:=\widehat{u}_{n, m}-\frac{\mathrm{i} \Delta t}{2\left(1+\xi_{1}^{2}\right)}\left(\xi_{1} \frac{\widehat{u_{n+1, r}^{p+1}}}{p+1}+\left(\xi_{1}+a \xi_{2}^{2} / \xi_{1}+b \xi_{3}^{2} / \xi_{1}\right) \widehat{u}_{n+1, r}+\xi_{1} \frac{\widehat{u_{n, m}^{p+1}}}{p+1}\right. \\
\left.+\left(\xi_{1}+a \xi_{2}^{2} / \xi_{1}+b \xi_{3}^{2} / \xi_{1}\right) \widehat{u}_{n, m}\right) .
\end{array}
$$

The iterations are stopped in one of the two following cases:

- when $\left\|u_{n+1, r+1}-u_{n+1, r}\right\|_{l^{2}(\Omega)} /\left\|u_{n+1,0}\right\|_{l^{2}(\Omega)} \leq \tau$, with $\tau>0$ a fixed tolerance. We then set $u_{n+1}:=u_{n+1, r+1}$;

- or when $r=m-1$. Here, we set $u_{n+1}:=u_{n+1, m}$. We mention that the step $\Delta t$ can be reduced in this case, in order to improve the previous relative error. 
This algorithm provides the solution $\widehat{u}_{n}\left(\xi_{1}, \xi_{2}, \xi_{3}\right)$, where $\xi_{1} \neq 0, \xi_{2}, \xi_{3}$ are defined as in (7), and where we consider:

- For $n=1,2, \ldots$,

$$
\widehat{u}_{n}\left(0, \xi_{2}, \xi_{3}\right):=\widehat{u}_{0}\left(0, \xi_{2}, \xi_{3}\right) \text {. }
$$

Remark 1.1 A practical way to choose a suitable (initial) step $\Delta t$ in the previous algorithm is the one involving the parameters $\Delta y, \Delta z$ as follows: for $\Omega=[-K, K] \times[-L, L] \times[-M, M]$,

$$
\Delta t<\frac{4}{1+(K / \Delta y)^{2}+(K / \Delta z)^{2}+\left\|u_{n}\right\|_{L^{\infty}(\Omega)}^{p}} .
$$

Indeed, since from (8),

$$
\begin{aligned}
\widehat{u}_{n+1, r+1}-\widehat{u}_{n+1} \approx & \mathrm{i} \frac{\Delta t}{2\left(1+\xi_{1}^{2}\right)}\left(\xi_{1}+a \xi_{2}^{2} / \xi_{1}+b \xi_{3}^{2} / \xi_{1}\right)\left(\widehat{u}_{n+1, r}-\widehat{u}_{n+1}\right) \\
& +\mathrm{i} \xi_{1} \frac{\Delta t}{2\left(1+\xi_{1}^{2}\right)}\left(\frac{\widehat{u_{n+1, r}^{p+1}}}{p+1}-\frac{\widehat{u_{n+1}^{p+1}}}{p+1}\right)
\end{aligned}
$$

it follows in the case where $p \in \mathbb{N}^{*}$ that,

$$
\begin{aligned}
\widehat{u}_{n+1, r+1}-\widehat{u}_{n+1} \approx & \mathrm{i} \frac{\Delta t}{2\left(1+\xi_{1}^{2}\right)}\left(\xi_{1}+a \xi_{2}^{2} / \xi_{1}+b \xi_{3}^{2} / \xi_{1}\right)\left(\widehat{u}_{n+1, r}-\widehat{u}_{n+1}\right) \\
& +\mathrm{i} \xi_{1} \frac{\Delta t}{2\left(1+\xi_{1}^{2}\right)(p+1)} \sum_{j=0}^{p}\left(u_{n+1, r}-\widehat{\left.u_{n+1}\right)} u_{n+1, r}^{j} u_{n+1}^{p-j},\right.
\end{aligned}
$$

and a linearization $\left(\right.$ near $\left.u_{n}\right)$ in the right-hand side of this relation leads to the approximation:

$\widehat{u}_{n+1, r+1}-\widehat{u}_{n+1} \approx \mathrm{i} \frac{\Delta t}{2\left(1+\xi_{1}^{2}\right)}\left(\xi_{1}+a \xi_{2}^{2} / \xi_{1}+b \xi_{3}^{2} / \xi_{1}\right)\left(\widehat{u}_{n+1, r}-\widehat{u}_{n+1}\right)+\mathrm{i} \xi_{1} \frac{\Delta t}{2\left(1+\xi_{1}^{2}\right)}\left(u_{n+1, r}-u_{n+1}\right) u_{n}^{p}$.

Then, since $\xi_{1} /\left(1+\xi_{1}^{2}\right) \leq 1 / 2, \xi_{2} \leq \pi / \Delta y, \xi_{3} \leq \pi / \Delta z$, it follows that for $k \neq 0$, and making use of the Parseval formula,

$$
\left\|u_{n+1, r+1}-u_{n+1}\right\|_{L^{2}(\Omega)} \lesssim \frac{\Delta t}{4}\left(1+(K / \Delta y)^{2}+(K / \Delta z)^{2}+\left\|u_{n}\right\|_{L^{\infty}(\Omega)}^{p}\right)\left\|u_{n+1, r}-u_{n+1}\right\|_{L^{2}(\Omega)} .
$$

\section{Long time behavior for localized initial data}

We are interested here in numerical simulations concerning the Cauchy problem. In a general way, two sets of parameters will be considered for these simulations based on the algorithm described previously. The first set is made up of the parameters $a, b= \pm 1, p \geq 1$, that intervene in (3), and the second one concerns the parameters $K, L, M, \Delta t, N_{x}, N_{y}, N_{z}$ used in the algorithm. Here, we focus on integer values of $p$, the numerical scheme proposed here works also for real and very large non-linearities.

To study the behavior in long time of the discrete solution of (3), associated with a localized initial datum, two phenomena will systematically be inspected: the dispersion (context where the $L^{\infty}$-norm of the solution decreases) and the solitonic behavior (phenomenon where the $L^{\infty}$-norm of the solution is constant). Let us remind that no theoretical result concerning the Cauchy problem based on (3) is established. The two-dimensional case of the numerical investigation regarding (3) is treated in [9]. 
Our numerical inspections will be based on the choice: for $\alpha, s \in \mathbb{R}_{+}^{\star}$,

$$
u_{0}(x, y, z)=\alpha\left(1-2 s x^{2}\right) \mathrm{e}^{-s\left(x^{2}+y^{2}+z^{2}\right)},
$$

and on various considerations of parameters mentioned above. Let us indicate that the choices of values of $K$ will be such that $u_{0}$ satisfies (5) numerically.

In the presentation of our results, we distinguish the case $p=1$ and the generalized case where $p>1$.

\section{$2.1 \quad$ Non-generalized case: $p=1$}

In this subsection, we describe the results obtained from numerical simulations by fixing $p=1$. In each of the contexts where $a= \pm 1$ with $b= \pm 1$, we perform experiments by setting here: $s=1, K=50$, $L=M=4, N_{y}=N_{z}=16$. We already mention that for all our experiments, here and in the next sections, the step $\Delta t$ will always be chosen in accordance with (9). We observe the same evolutions of these norms when $a=-1, b=1$ as well as when $a=1, b=-1$. This was foreseeable according to the symmetry of $u_{0}$ and to the one of (3) related to the $y$ and $z$-directions. In this order of ideas, we restrict in this section the presentation of our results to the contexts where $a b=1$, and $a=-1, b=1$.

The results represented in Figure 1 concern the evolutions with respect to time of the $l^{\infty}$ and $h^{1}$-norms of the discrete solution obtained by considering $N_{x}=256, \Delta t=10^{-3}, \alpha=5$.
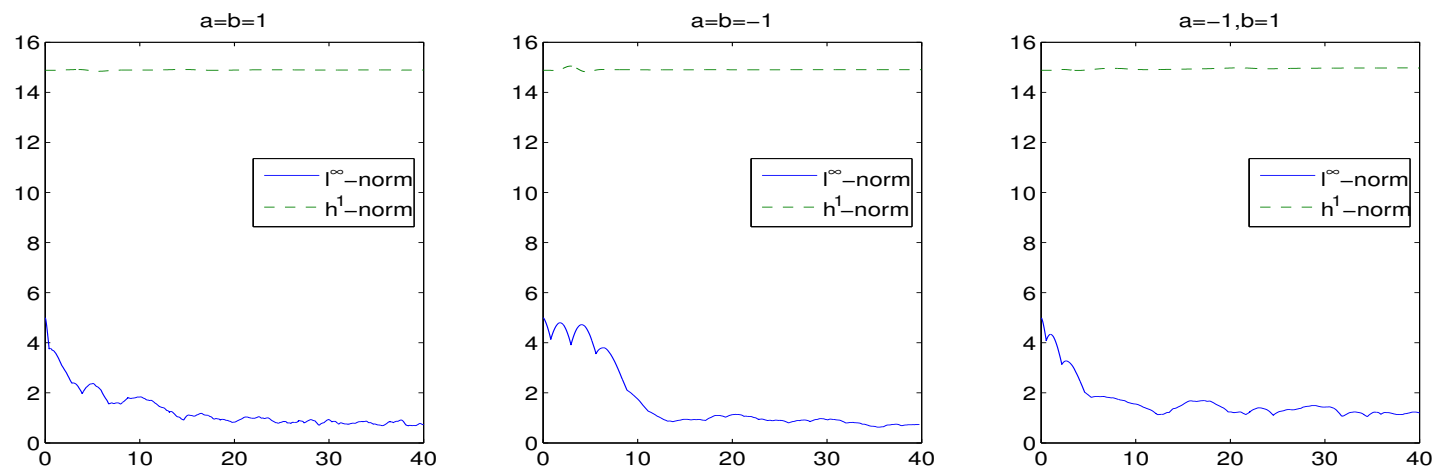

Figure 1: Evolutions of the $h^{1}$-norm (---) and the $l^{\infty}$-norm (-) with respect to time, for $p=1, \alpha=$ $5, s=1, K=50, L=M=4, N_{x}=256, N_{y}=N_{z}=16, \Delta t=10^{-3}$.

Let us notice that for these choices of $\alpha$, the results obtained from simulations with other values of parameters, namely with $K=32, L=M=6,12, N_{x}=128, N_{y}=N_{z}=32,64$, do not qualitatively differ from those represented in this section. We observe that the $h^{1}$-norm is conserved by the flow of (3), and the $l^{\infty}$-norm decreases with time. Put another words, the dispersive effect prevails over the non-linearity. The evolutions of the norms in the different contexts where $a b= \pm 1$ look similar.

Figure 2 presents the evolutions of the $l^{\infty}$ and $h^{1}$-norms of the discrete solution when a larger amplitude of the initial datum is considered. Here $\alpha=50, N_{x}=512$ and $\Delta t=10^{-4}$.

In each of the contexts where $a=b=1, a b=-1$, we observe, as with $\alpha=5$, that the dispersive effect prevails over the non-linearity (similar to Figure 1). But for $a=b=-1$, the discrete solution, associated with the initial datum of larger amplitude, has a tendency to blow up at the beginning of the evolution and after that, the dispersive effect prevents the solution from blowing up. The fact that the non-linearity and the dispersive effect are neutralized, and that the $L^{\infty}$-norm is stabilized, is called the solitonic behavior. This means that the velocity of the limit profile has a periodic-like behavior in time. We mention that when we take larger values of $L, M, N_{y}, N_{z}$ (e.g. $L=M=6, N_{y}=N_{z}=32$ ), we do not obtain, from the simulations, results that are qualitatively different from those presented here. 

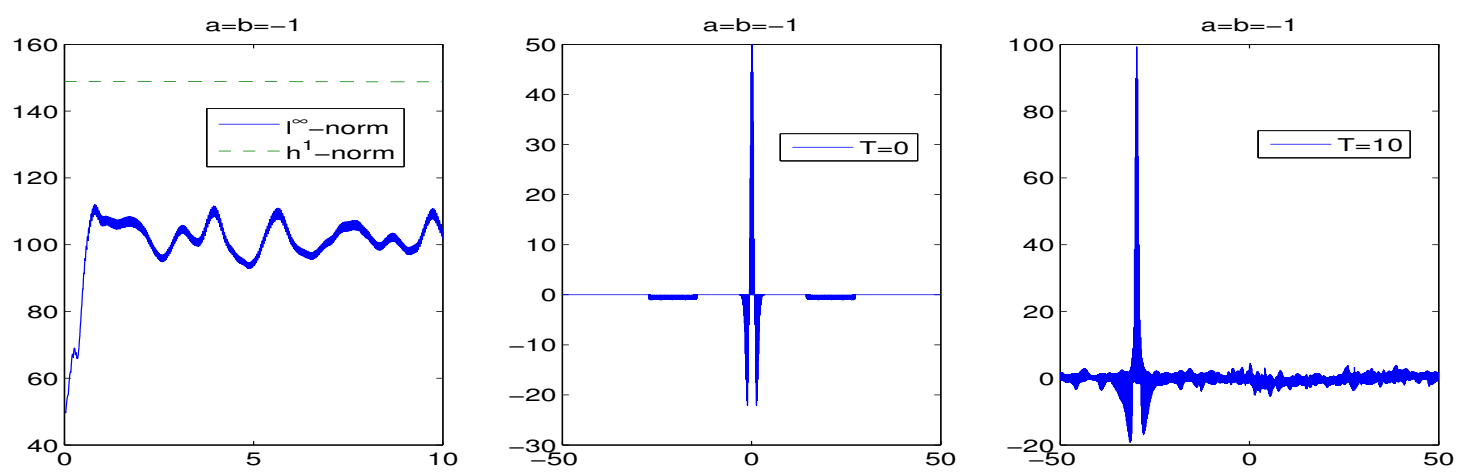

Figure 2: At left, evolutions of the $h^{1}$-norm (-- ) and the $l^{\infty}$-norm $(-)$ with respect to time. At center, the projection on the $x$-axis of the localized initial datum. At right, the projection at time $T=10$. Here $p=1, a=b=-1, \alpha=50, s=1, K=50, L=M=4, N_{x}=512, N_{y}=N_{z}=16, \Delta t=10^{-4}$.

\subsection{Generalized case: $p>1$}

In this subsection, we are concerned with numerical experiments when $p=2,3$.

Let us fix in this part $p=2$. The experiments associated with Figure 3 is performed by setting: $s=1$, $K=50, L=M=4, N_{x}=512, N_{y}=N_{z}=16$ and $\Delta t=10^{-4}$. In Figure 3, we represent the evolutions with respect to time of the $l^{\infty}$ and $h^{1}$-norms of the discrete solution obtained by considering $\alpha=10$.
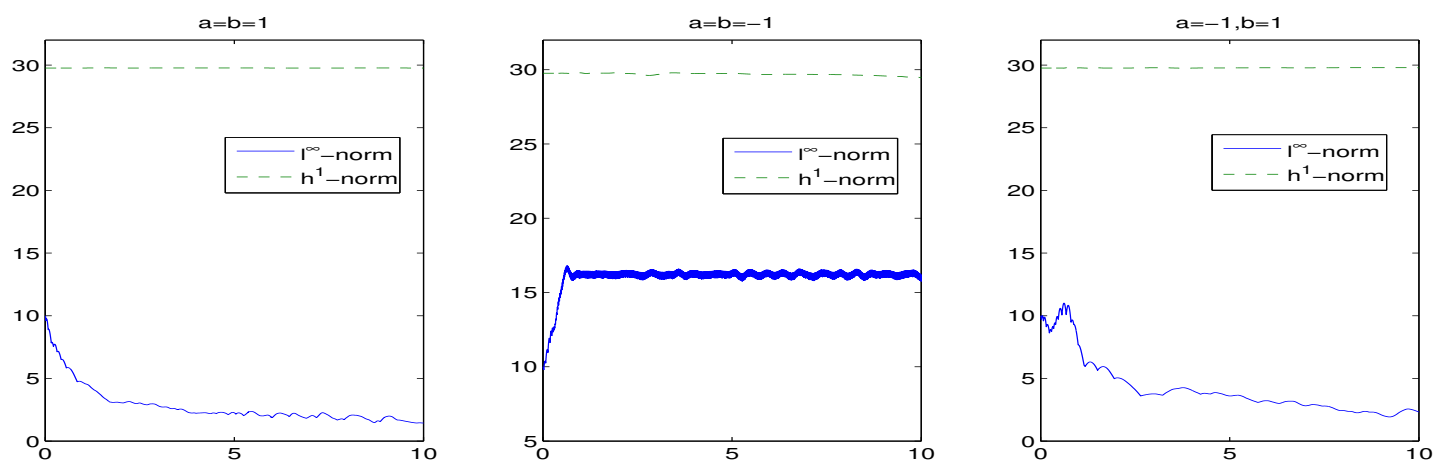

Figure 3: Evolutions of the $h^{1}-$ norm (--) and the $l^{\infty}$-norm (-) with respect to time, for $p=2, \alpha=$ $10, s=1, K=50, L=M=4, N_{x}=512, N_{y}=N_{z}=16, \Delta t=10^{-4}$.

It appears that the $l^{\infty}$-norm has a tendency to blow up and then the evolution of the norm is stabilized, the solitonic behavior is observed. As for the case $p=1$, we observe that the dispersive effect prevails over the non-linearity in the contexts where $a=b=1, a b=-1$.

The numerical results known from [10] concerning the equation (1), for $p=2$, in the context where $a=b=-1$, report that the non-linearity is stronger than the dispersive effect and the solution blows up. The behavior of the solution is here completely different, the solitonic behavior is observed. When $a=b=1$ and $a b=-1$, the dispersion is also observed for solutions of (1).

The observations are confirmed when we use larger values of $N_{x}, N_{y}, N_{z}$ (e.g. with $N_{x}=1024, N_{y}=$ $\left.N_{z}=32\right)$.

We can already predict that, since the non-linearity was persistent with $p=2$, the solitonic behavior 
will be at least observed in the case $p=3$ because of a stronger non-linearity.

Let us now fix $p=3$. In our first experiments, associated with Figure 4, we considered $s=1$, $L=M=6, N_{x}=512, N_{y}=N_{z}=32$ and $\Delta t=10^{-4}$. Figure 4 shows the evolutions (with respect to time) of the $l^{\infty}$ and $h^{1}$-norms of the discrete solution obtained when $K=50, \alpha=5$.
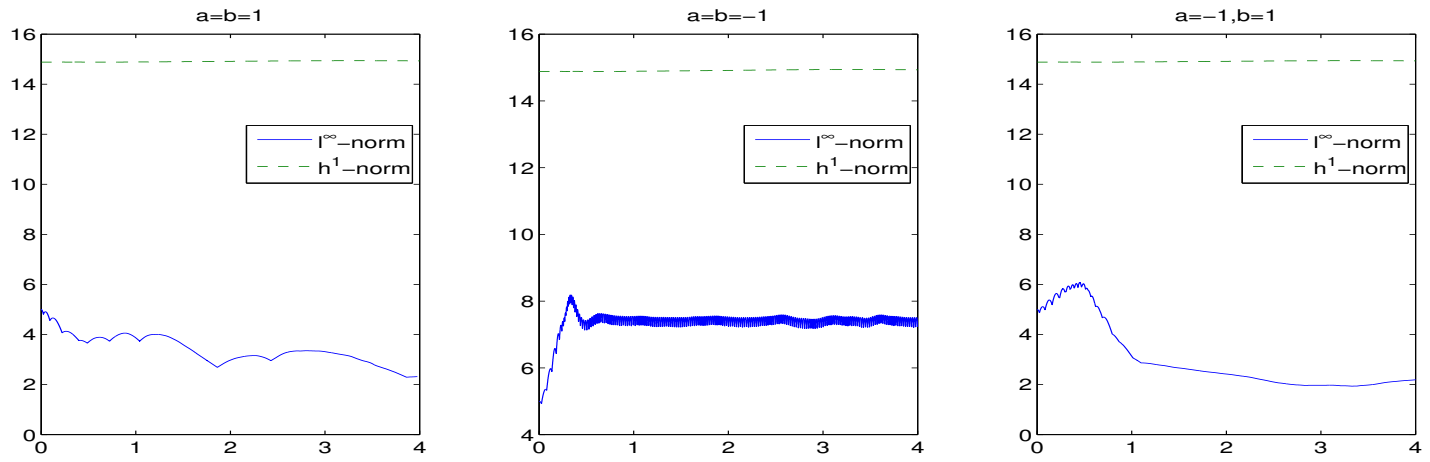

Figure 4: Evolutions of the $h^{1}$-norm (---) and the $l^{\infty}$-norm (-) with respect to time, for $p=3, \alpha=$ $5, s=1, K=50, L=M=6, N_{x}=512, N_{y}=N_{z}=32, \Delta t=10^{-4}$.

These experiments confirm that the non-linearity effect is effectively as strong as the dispersive effect. When $a=b=-1$, the solution has a tendency to blow-up at the begining and then is stabilized. In the other hand, the solution slowly decreases, then the $L^{\infty}$-norm is also stabilized (in Figure $4, l^{\infty}$-norm remains close to 2 at left and right).

Let us now summarize our results. The effect of dispersion are less important for two negative transverse directions than for two positive or opposite transverse directions. Indeed, the solitonic behavior of the discrete solution, in the context where $a=b=-1$, is observed when $p \geq 1$, whereas the one involved in each of the contexts where $a=b=1$, and $a b=-1$ when $p=3$. Moreover, comparing to the result observed for the solutions of (1), instead of the blow-up in finite time [10], the solitonic behavior takes place.

Let us notice that the solitonic behavior observed in this paper is not in contradiction with the nonexistence results of solitary waves in [6]. In every cases, we obtain a destabilized solitonic behavior in the sense that the amplitude of the solution varies not much but the solution do not remain close to the initial datum. In other words, there exists $T>0$ such that for $t \geq T$

$$
\|u(T)-u(t)\|_{\infty} \leq \epsilon, \text { and }\left\|u_{0}(.-c t)-u(t)\right\|_{\infty} \geq C .
$$

\section{Transverse instabilities in the $y$ and $z$-directions}

The generalized equation (3) admits as solution the line-soliton

$$
\Phi_{c}(x, t)=\left(\frac{(p+1)(p+2)}{2} c\right)^{1 / p} \operatorname{sech}^{2 / p}\left(\frac{p}{2}(x-c t)\right),
$$

which is a solution of the generalized BBM equation. This represents a solitary wave, moving from left to right in the $x$-direction, at velocity $c$, without deformation. In this section, we study the evolution in time of this line-soliton perturbed in an infinitesimal way in the two transverse $y$ and $z$-directions. We consider then the Cauchy problem by defining the initial datum $u_{0}$ as follows,

$$
u_{0}(x, y, z):=\Phi_{c(y, z)}(x, 0),
$$


where $c(y, z):=c\left(1+\varepsilon \cos \left(2 \pi y / \lambda_{y}+2 \pi z / \lambda_{z}\right)\right)$, with $\varepsilon>0$ a fixed small value; this perturbed velocity is not uniform in the transverse directions, $\lambda_{y}$ and $\lambda_{z}$ are the wavelengths.

\subsection{Non-generalized case: $p=1$}

We fix $p=1$ in this subsection. In addition to the two previous sets of parameters, our numerical experiments will be performed with respect to diverse considerations of $c, \lambda_{y}$ and $\lambda_{z}$.

In each of the contexts where $a b= \pm 1$, we perform simulations by fixing $K=50, L=M=4$, $N_{x}=256, N_{y}=N_{z}=16, \Delta t=10^{-3}, \varepsilon=10^{-2}$. Results are not influenced by the choice of smaller steps, and of smaller values of $\varepsilon$.

We start by considering the context where $a=b=-1$. In Figure 5 , we represent the evolutions of the $l^{\infty}$ and $h^{1}$-norms of the discrete solution obtained respectively with $\lambda_{y}=\lambda_{z}=50$, for $c=4$.
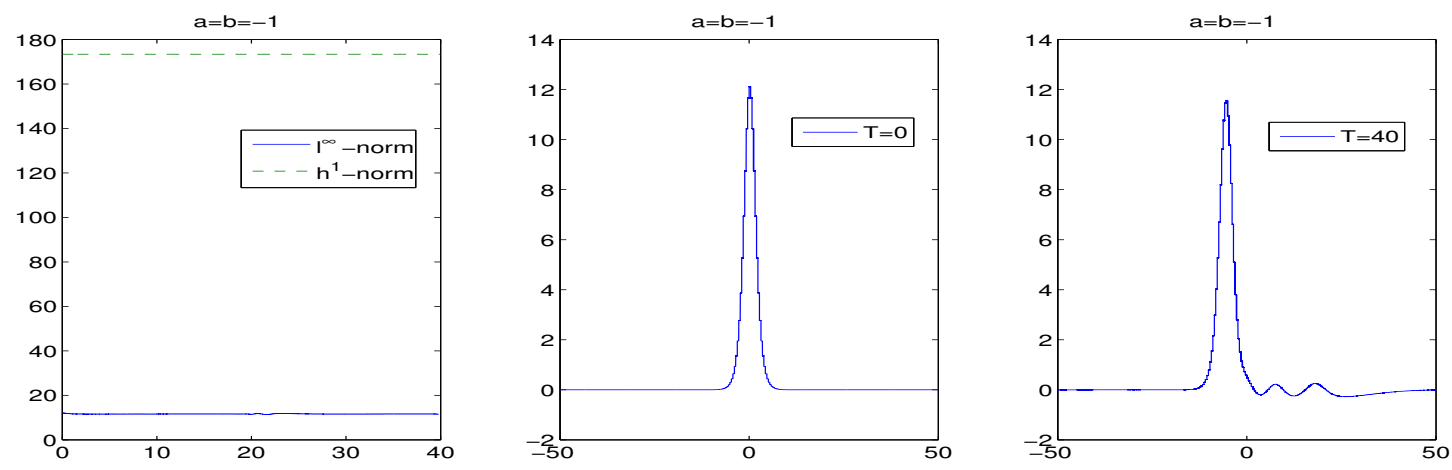

Figure 5: At left, evolutions of the $h^{1}$-norm (--) and the $l^{\infty}$-norm (-) with respect to time, for $p=1, a=b=-1, c=4, K=50, L=M=4, N_{x}=256, N_{y}=N_{z}=16, \Delta t=10^{-3}$. Here, $\lambda_{y}=\lambda_{z}=50$ and $\varepsilon=10^{-2}$. At center, the projection on the $x$-axis of the initial line-soliton. At right, the projection at time $T=40$.

Similar results derive also from simulations by using larger values for $K, L, M, N_{x}, N_{y}, N_{z}$, and by considering distinct values for $\lambda_{y}$ and $\lambda_{z}$.

Let us now consider the contexts where $a b=-1$, by using again $c=4$ in the experiments. Figure 6 shows the results obtained respectively for $a=-1, b=1$, when $\lambda_{y}=0.01, \lambda_{z}=50$.
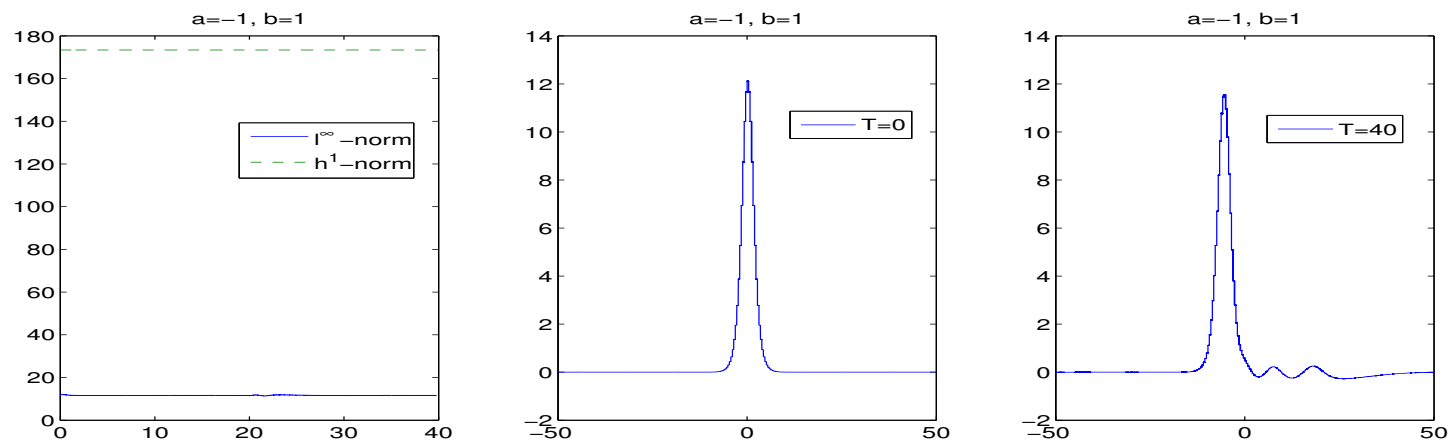

Figure 6: At left, evolutions of the $h^{1}-$ norm (---) and the $l^{\infty}$-norm $(-)$ with respect to time. At center, the projection on the $x$-axis of the initial line-soliton. At right, the projection at time $T=40$. Here $p=1, a=1, b=-1, c=4, K=50, L=M=4, N_{x}=256, N_{y}=N_{z}=16, \Delta t=10^{-3}, \lambda_{y}=0.01, \lambda_{z}=50$ and $\varepsilon=10^{-2}$. 
We observe from simulations the same evolutions of the norms in the contexts where $a b= \pm 1$. The profile of the discrete solution is slightly perturbed during time, and the amplitude of wave remains close to the one of the initial datum. Some dispersive tail appears and the number of waves is increasing with respect to time.

\subsection{Generalized case: $p>1$}

We aim here to study the transverse instabilities in the case where $p=2$.

Our experiments are performed in each of the contexts where $a b= \pm 1$, by fixing $K=50, L=M=4$, $N_{x}=256, N_{y}=N_{z}=16$ and $\Delta t=5 \times 10^{-4}, \varepsilon=10^{-2}$. Figures 7 and 8 present, for $c=4$, the evolutions of the $h^{1}$ and $l^{\infty}$-norms of the discrete solution obtained respectively with $\lambda_{y}=0.01,50, \lambda_{z}=50$.
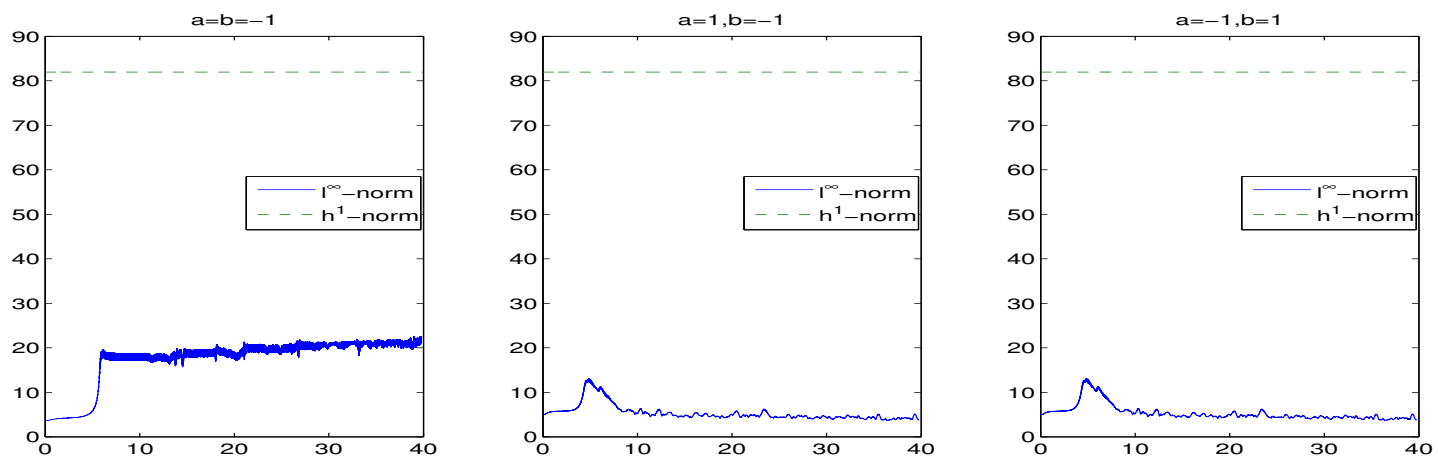

Figure 7: Evolutions of the $h^{1}$-norm (--) and the $l^{\infty}$-norm (-) with respect to time for $p=2, a=$ $b=-1, a b=-1, c=4, K=50, L=M=4, N_{x}=256, N_{y}=N_{z}=16, \Delta t=5 \times 10^{-4}, \lambda_{y}=\lambda_{z}=50$ and $\varepsilon=10^{-2}$
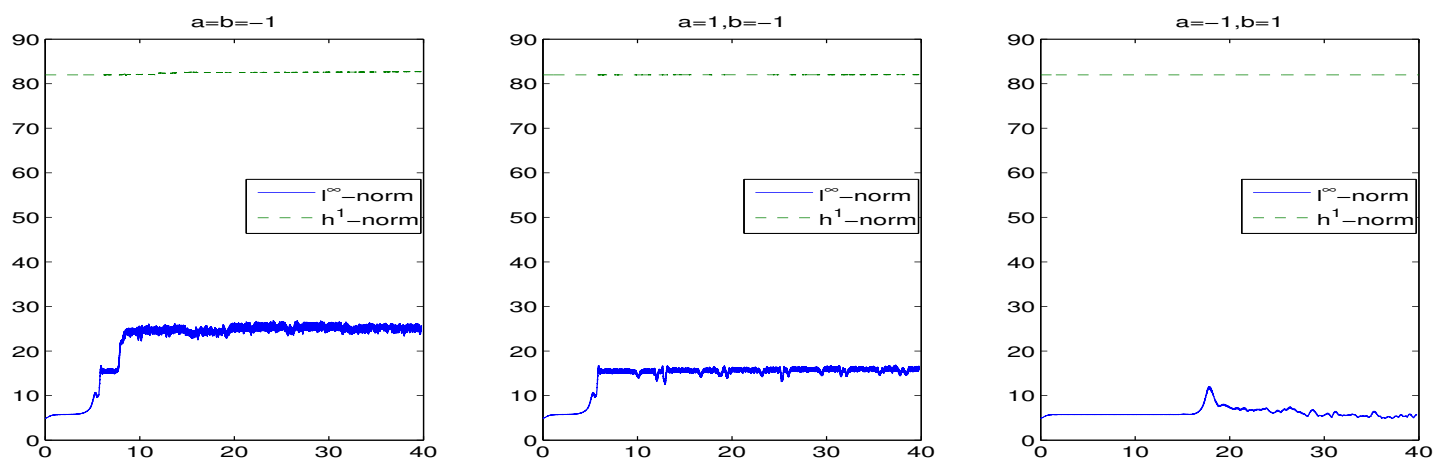

Figure 8: Evolutions of the $h^{1}$-norm (-- ) and the $l^{\infty}$-norm $(-)$ with respect to time. Here $p=2, a=$ $b=-1, a b=-1, c=4, K=50, L=M=4, N_{x}=256, N_{y}=N_{z}=16, \Delta t=5 \times 10^{-4}, \lambda_{y}=0.01, \lambda_{z}=50$ and $\varepsilon=10^{-2}$.

In the contexts $a=b=-1$ and $a b=-1$, the solitonic behavior is observed. When $a=b=1$, we obtain similar results as in Figure 5.

We can inspect the influence of the velocity. Due to the non-homogeneous dependence of the velocity, it is difficult to determine a precise critical value. Independently of the small values of $\varepsilon$, we notice that 
the larger is the velocity, the smaller is this critical value. For $c>0$, there exists a critical transverse wavelength $\lambda_{c}^{1}=\lambda^{1} / c$ such that if $\lambda_{y}$ and $\lambda_{z}$ is larger than $\lambda_{c}^{1}$, the perturbed line-soliton is unstable.

We extend our experiments to very large wavelengths. We can notice that the formal limit of the perturbed line-soliton, when $\lambda_{y}$ and $\lambda_{z}$ tend to infinity, gives $\Phi_{c(1+\varepsilon)}$, which does not depend on the transverse variables. In particular, the solution of (3) with $\Phi_{c(1+\varepsilon)}$ as initial datum is stable [29]. We thus deduce, in the context where $a=b=-1$, that there exists another critical transverse wavelength $\lambda_{c}^{2}$ such that if $\lambda_{y}$ or $\lambda_{z}>\lambda_{c}^{2}$, the perturbed line-soliton is no longer unstable (similar to Figure 5).

The results are confirmed by the case $p=3$. Even in the context $a=b=-1$ and $a b=-1$, the discrete solution is unstable.
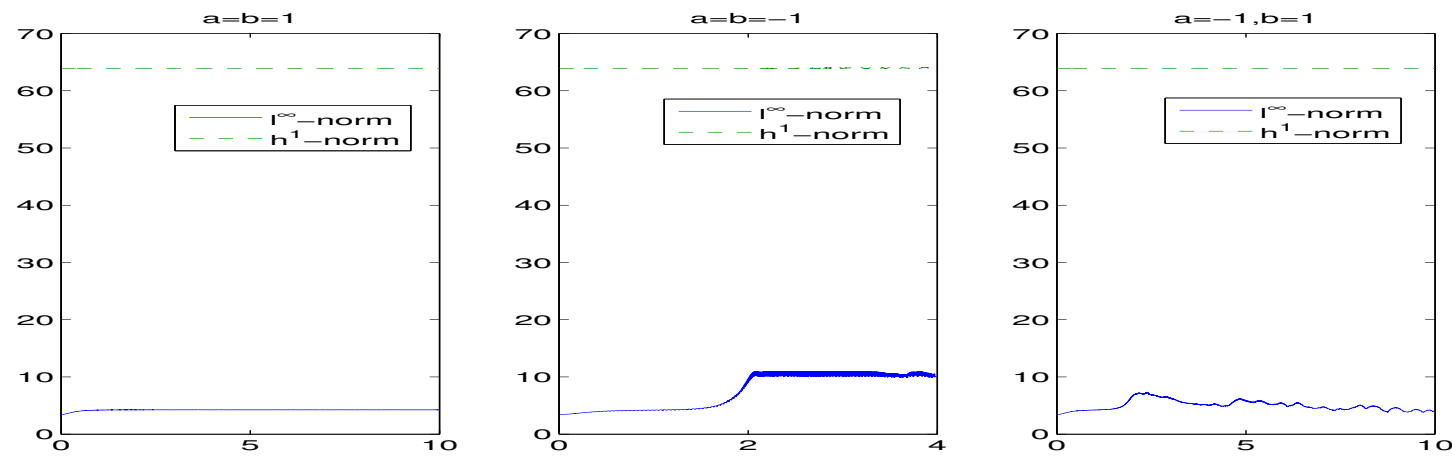

Figure 9: Evolutions of the $h^{1}-$ norm (-- ) and the $l^{\infty}$-norm $(-)$ with respect to time. Here $p=3, c=$ $4, K=50, L=M=6, N_{x}=512, N_{y}=N_{z}=32, \Delta t=10^{-4}, \lambda_{y}=\lambda_{z}=50$ and $\varepsilon=10^{-2}$.

Let us now summarize the numerical observations of this section. When $p=1$ and $a=b=-1, a b=$ -1 or $p \geq 1, a=b=1$, the profile of the discrete solution remains close to the initial datum and some dispersive tail appears and increases with respect to time. This does not allow to conclude on the stability of the line-soliton, the range of perturbations being not sufficiently exhaustive.

The experiments for $p=2,3$ show that when the line-soliton is perturbed in the two transverse directions by neither very small nor very large wavelengths, the associated discrete solution is unstable in the sense that the solution does not remain close to the initial datum but behaves like a soliton. Concerning the KP equations (1), the solution is also unstable, and moreover the solution blows up in finite time [10].

These observations lead us to formulate the following conjecture.

\section{Conjecture 3.1}

For $\varepsilon>0$ sufficiently small, let $u_{0}$ be the perturbed line-soliton of velocity $c(y, z):=c\left(1+\varepsilon \cos \left(2 \pi y / \lambda_{y}+\right.\right.$ $\left.2 \pi z / \lambda_{z}\right)$ ), where $\lambda_{y}, \lambda_{z}>0$. Let $p \geq 2$, and $a=b=-1$ or $a b=-1$. There exist two critical transverse wavelengths $0<\lambda_{c}^{1} \ll \lambda_{c}^{2}$, depending only on $c$, such that the solution $u$ of (3), with $u_{0}$ as initial datum, is unstable under the flow of (3) if $\lambda_{c}^{1}<\lambda_{y}<\lambda_{c}^{2}$ respectively or $\lambda_{c}^{1}<\lambda_{z}<\lambda_{c}^{2}$.

\section{Transverse instability in the $z$-direction}

We are concerned in this section with the perturbation of the profile of the Zaitsev traveling waves [31]. The function

$$
\psi_{c}(x, y)=12 \alpha^{2} c \frac{1-\beta \cosh (\alpha x) \cos (\delta y)}{(\cosh (\alpha x)-\beta \cos (\delta y))^{2}}
$$


with

$$
\beta=\sqrt{1-\frac{3 c \alpha^{4}}{\delta^{2}}}, 3 c \alpha^{4}<\delta^{2}, \alpha^{2}+\frac{\delta^{2}}{c \alpha^{2}}=1, p=1, a=-1
$$

which is localized in the $x$-direction and periodic in the $y$-direction, represents such a profile.

We want to study the evolution, subject to (3), of the Zaitsev wave when it is perturbed in the second transverse direction. We then consider

$$
u_{0}(x, y, z):=\psi_{c(z)}(x, y, 0),
$$

as the initial datum associated with (3). The velocity is here slightly perturbed in the $z$-direction; namely, $c(z):=c\left(1+\varepsilon \cos \left(2 \pi z / \lambda_{z}\right)\right)$, with $\varepsilon>0$ small and $\lambda_{z}>0$ a wavelength.

Our numerical simulations will be performed in each of the contexts where $b= \pm 1$. In what follows, we consider $K=50, L=12, M=4, N_{x}=256, N_{y}=32, N_{z}=16, \Delta t=10^{-3}, \varepsilon=10^{-2}$. The value of $L$ is larger than in the previous sections in such a way that the present initial datum $u_{0}$ also satisfies (5) numerically.

The results represented in Figure 10 concern the context where $b=-1$, and show for $c=4$ the evolutions of the $h^{1}$ and $l^{\infty}$-norms of the discrete solution obtained with $\lambda_{z}=50$. Similar results derive from simulations, even by using larger values of $K, L, N_{x}, N_{y}$ and smaller values of $\varepsilon$.
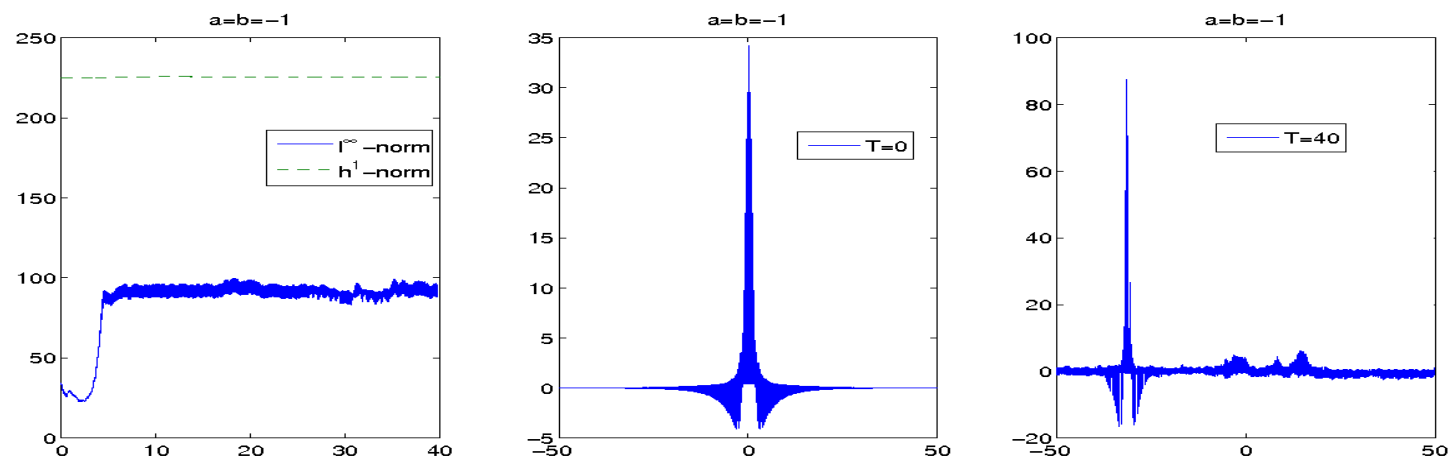

Figure 10: At left, evolutions of the $h^{1}$-norm (---) and the $l^{\infty}$-norm (-) with respect to time. At center, the projection on the $x$-axis of the initial Zaitsev soliton. At right, the projection at time $T=40$. Here $b=-1, c=4, K=32, L=12, M=4, N_{x}=256, N_{y}=32, N_{z}=16, \Delta t=10^{-3}, \lambda_{z}=50$ and $\varepsilon=10^{-2}$.

We notice that there exists a critical transverse wavelength $\lambda_{c}^{1}=\lambda^{1} / c$, such that if $\lambda_{z}>\lambda_{c}^{1}$, the solution has a tendency to blow up and then is stabilized. Moreover, there exists another critical transverse wavelength $\lambda_{c}^{2}$ such that if $\lambda_{z}>\lambda_{c}^{2}$, the perturbed Zaitsev wave is no more unstable.

In the last context where $b=1$, the results of Figure 11 are obtained from experiments performed in this context by setting $c=4$. The solution is also unstable, but in this case, no solitonic behavior is observed. 

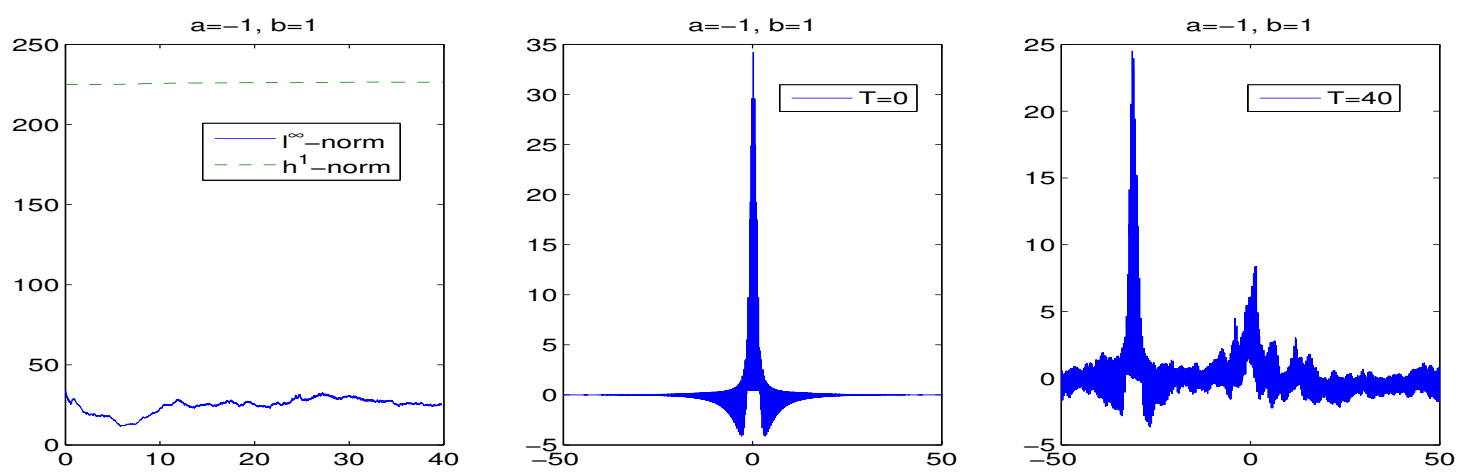

Figure 11: At left, evolutions of the $h^{1}$-norm (---) and the $l^{\infty}$-norm $(-)$ with respect to time, for $b=1, c=4, K=50, L=12, M=4, N_{x}=256, N_{y}=32, N_{z}=16, \Delta t=10^{-3}$. Here, $\lambda_{z}=50$ and $\varepsilon=10^{-2}$. At center, the projection on the $x$-axis of the initial Zaitsev soliton. At right, the projection at time $T=40$.

It follows that the wavelength $\lambda_{z}$ characterizes the instability of the Zaitsev wave by transverse perturbations in the $z$-direction. More precisely, when $b= \pm 1$, there exist two critical transverse wavelengths $0<\lambda_{c}^{1} \ll \lambda_{c}^{2}$, depending only on $c$, such that the corresponding discrete solution is unstable if $\lambda_{c}^{1}<\lambda_{z}<\lambda_{c}^{2}$.

Then, we can formulate the following conjecture.

\section{Conjecture 4.1}

For $\varepsilon>0$ sufficiently small, let $u_{0}$ be the perturbed Zaitsev wave of velocity $c(z):=c\left(1+\varepsilon \cos \left(2 \pi z / \lambda_{z}\right)\right)$, where $\lambda_{z}>0$. Let $p=1$, and $a=-1$. There exist two critical transverse wavelengths $0<\lambda_{c}^{1} \ll \lambda_{c}^{2}$, depending only on $c$, such that the solution of (3), with $u_{0}$ as initial datum, is unstable if $\lambda_{c}^{1}<\lambda_{z}<\lambda_{c}^{2}$.

\section{References}

[1] J.C. Alexander, R.L. Pego \& R.L. Sachs, On the transverse instability of solitary waves in the Kadomtsev-Petviashvili equation, Phys. Lett. A, A 226, (1997), 187-192.

[2] T.B. Benjamin, The stability of solitary waves, Proc. Roy. Soc. Lond., Ser. A, 328, no. 1573, (1972), $153-183$.

[3] T.B. Benjamin, J.L. Bona \& J.J Mahony, Model equations for long waves in nonlinear dispersive systems, Phil. Trans. R. Soc. London, Ser. A, 272, (1972), 47-78.

[4] J.L. Bona, Y. Liu, \& M.M Tom, The Cauchy problem and stability of solitary-wave solutions for RLW-KP-type equations, J. Diff. Eq., 185, no. 2, (2002), 437-482.

[5] F. Bethuel, P. Gravejat \& J.-C. Saut, Existence and properties of travelling waves for the Gross-Pitaevskii equation, Cont. Math., 473, (2008), 55-104.

[6] A. De Bouard \& J.-C. Saut, Solitary waves of generalized Kadomtsev-Petviashvili equations, Ann. I.H.P., Anal. Non Lin., 4, (1997), 211-236.

[7] A. De Bouard \& J.-C. Saut, Symmetries and decay of the generalized Kadomtsev-Petviashvili solitary waves, SIAM J. Math. Anal., 28,5, (1997), 1064-1085.

[8] G.E. Falkovitch \& S.K. Turitsyn, Stability of magnetoelastic solitons and self-focusing of sound in antiferromagnet, Sov. Phys. JETP, 62, (1985), 146-152.

[9] F. Hamidouche, Simulations numériques des équations de Kadomtsev-Petviashvili, Ph. D. Thesis, Université Paris-Sud, 2001.

[10] F. Hamidouche, Y. Mammeri \& S.M. Mefire, Numerical study of solutions of the $3 D$ generalized KadomtsevPetviashvili equations for long times, CiCP, 6, no. 5, (2009), 1022-1062. 
[11] B.B. Kadomtsev \& V.I. Petviashvili, On the stability of solitary waves in weakly dispersing media, Soviet Physics Doklady, 15,6, (1970), 539-541.

[12] C.M. Khalique \& A.R. Adem, Exact solutions of a generalized (3+1)-dimensional Kadomtsev-Petviashvili equation using Lie symmetry analysis, Applied Mathematics and Computation, 216, no. 10, (2010), 2849-2854.

[13] C. Klein \& J.-C. Saut, Numerical study of blow up and stability of solutions of generalized Kadomtsev-Petviashvili equations, arXiv:1010.5510.

[14] H. Leblond, KP lumps in ferromagnets: a three-dimensional KdV-Burgers model, J. Phys. A, 35, no. 47, (2002), 10149-10161.

[15] Y. Liu, Strong instability of solitary-wave solutions to a Kadomtsev-Petviashvili equation in three dimensions, J. Diff. Eq., 180, (2002), 153-170.

[16] Y. Liu, Blow up and instability of solitary-wave solutions to a generalized Kadomtsev-Petviashvili equation, Trans. Amer. Math. Soc., 353, no. 1, (2000), 191-208.

[17] W.-X. Ma \& Z. Zhu, Solving the (3+1)-dimensional generalized KP and BKP equations by the multiple exp-function algorithm, Applied Mathematics and Computation, 218, no. 24, (2012), 11871-11879.

[18] A. Quarteroni, R. Sacco \& F. Saleri, Numerical mathematics, Springer-Verlag, New York, 2000.

[19] F. Rousset \& N. Tzvetkov, Transverse nonlinear instability of solitary waves for some hamiltonian PDEs, J. Math. Pures Appl., 90, no. 6, (2008), 550-590.

[20] F. Rousset \& N. Tzvetkov, Transverse nonlinear instability for two-dimensional dispersive models, Ann. I.H.P., Anal. non lin., 26, no. 2, (2009), 477-496.

[21] F. Rousset \& N. Tzvetkov, A simple criterion of transverse linear instability for solitary waves, Math. Res. Lett., 17, no. 1, (2010), 157-169.

[22] F. Rousset \& N. Tzvetkov, Transverse instability of the line solitary water waves, Invent. Math., 184, no. 2, (2011), $257-388$.

[23] F. Rousset \& N. Tzvetkov, Stability and instability of the KdV solitary wave under the KP-I flow, Commun. Math. Phys., 313, no. 1, (2012), 155-173.

[24] J.-C. Saut, Recent results on the generalized Kadomtsev-Petviashvili equations, Acta Appl. Math., 39, (1995), $477-487$.

[25] J.-C. Saut \& N.Tzvetkov, Global well-posedness for the KP-BBM equations, AMRX, 1, (2004), 1-16.

[26] S. M. El-Sayed \& D. Kaya, The decomposition method for solving (2+1)-dimensional Boussinesq equation and (3+1)dimensional KP equation, Applied Mathematics and Computation, 157, no. 2, (2004), 523-534.

[27] A.-M. Wazwaz, Exact solutions of compact and noncompact structures for the KP-BBM equation, Applied Mathematics and Computation, 169, no. 1, (2005), 700-712.

[28] A.-M. Wazwaz, A computational approach to soliton solutions of the Kadomtsev-Petviashvili equation, Applied Mathematics and Computation, 123, no. 2, (2001), 205-217.

[29] M.I. Weinstein, Existence and dynamic stability of solitary wave solutions of equations arising in long wave propagation, Comm. PDEs, 12, no. 10, (1987), 1133-1173.

[30] Y.-Q. Wu, Asymptotic behavior of the periodic wave solution for the (3+1)-dimensional Kadomtsev-Petviashvili equation, Applied Mathematics and Computation, 216, no. 11, (2010), 3154-3161.

[31] A.A. Zaitsev, Formation of stationary waves by superposition of solitons, Sov. Phys. Dokl., 28, no. 9, (1983), 720-722.

[32] H. Zhao, Interactions of solitary waves under the conditions of the (3+1)-dimensional Kadomtsev-Petviashvilli equation, Applied Mathematics and Computation, 215, no. 9, (2010), 3383-3389. 